Review

\title{
MicroRNAs associated with therapy of non-small cell lung cancer
}

\author{
Junmi Lu, Yuting Zhan, Juan Feng, Jiadi Luo, Songqing Fan ${ }^{\bowtie}$ \\ Department of Pathology, the Second Xiangya Hospital, Central South University, Changsha, Hunan, China \\ $\triangle$ Corresponding author: Songqing Fan, Department of Pathology, the Second Xiangya Hospital of Central South University, Changsha, Hunan, China. Email: \\ songqingfan@csu.edu.cn \\ (c) Ivyspring International Publisher. This is an open access article distributed under the terms of the Creative Commons Attribution (CC BY-NC) license \\ (https://creativecommons.org/licenses/by-nc/4.0/). See http://ivyspring.com/terms for full terms and conditions.
}

Received: 2017.08.05; Accepted: 2018.02.25; Published: 2018.03.10

\begin{abstract}
Background \& Objective: The incidence of non-small cell lung cancer (NSCLC) has been rising over the past several decades. Despite various therapeutic regimens and modern diagnostic techniques are developed, NSCLC still have an extremely poor prognosis due to drug resistance. Therefore, it is critical to find a novel precise diagnosis and effective treatment approach for NSCLC patients. MicroRNAs (MiRNAs) are a class of 18-25nt non-coding small RNAs, which have been shown to be involved profoundly in the pathogenesis such as cellular proliferation, differentiation, development, apoptosis and tumorigenesis in many human tumors including of NSCLC. We reviewed existing research literature regarding correlations between miRNAs and their target's response to anticancer treatment, and summarized the recent findings between miRNAs and therapy availability in NSCLC.
\end{abstract}

Key words: NSCLC, microRNAs, targeted therapy, radio-chemotherapy

\section{Introduction}

Lung cancer is one of the most common malignant tumors and has become the primary form of cancer-related death in the world. As one category of lung cancer, NSCLC concludes lung adenocarcinoma, lung squamous carcinoma and large cell lung cancer [1,2]. Although various therapies have been applied to clinical practice, patients still have poor five-year survival rates because of advanced period and resistance to therapies, which places a large health burden on society [3]. With both traditional radiochemical and novel targeted therapies, most initially sensitive patients will eventually develop a "secondary resistance" to these therapies.

MiRNAs are a class of $18-25 n t$ non-coding small RNAs without open reading frames (ORFs) in living species, which have been confirmed to be involved in multiple biological processes, such as carcinogenesis, angiogenesis, cell proliferation, invasion, migration, differentiation and apoptosis, and play crucial roles in the regulation of tumor development and progression
$[4,5]$. It is discovered that every mature miRNA originate from long primary microRNA (pri-miRNA) transcript or precursor microRNA (pre-miRNA) molecules, which are then sheared by biological enzymes Drosha and Dicer, and finally integrate to RNA induced silencing complex (RISC). Current studies show that if this miRISC is binding with targeted mRNA 3' UTR, the targeted mRNAs translation might be inhibited in some degree [5]. These features have been regarded as a novel idea in the future therapy of malignant tumors. However, the distribution of miRNAs might indicate functional specificity in vivo and vitro.

Interestingly, many miRNAs are overexpressed in NSCLC tissues, but have low expression in the adjacent normal tissues, called proto-oncogenes. Additionally, several miRNAs are found to be under-expressed in the NSCLC tissues, which are considered as "anti-oncogenes" $[6,7,8]$. Furthermore, miRNAs which target multiple genes and different groups of target genes of the same miRNA are often 
regulated mutually. Therefore, some genes can also be co-targeted by different miRNAs, which form a dedicated regulatory net (Figure 1) [8]. Based on the research mentioned above, miRNAs might serve as novel and promising potential biomarkers for diagnosis, prognosis and therapy of patients with NSCLC.

\section{MiRNAs and sensitivity to therapy of NSCLC}

\section{MiRNAs and sensitivity to targeted therapy of NSCLC}

New targeted cancer therapies will increasingly replace radio-chemotherapy because of considering many physical, chemical and biological damages caused by radioactive rays [9]. Compared with traditional radio-chemotherapy, novel targeted therapies feature more specificity and efficiency in the cancer therapy. Binding to ATP-binding sites of epidermal growth factor receptor-tyrosine kinase (EGFR-TK) domain and its downstream signal pathways, such as epidermal growth factor receptor/phosphoinositide-3 kinase/protein kinase-B / mammalian target of rapamycin (EGFR/PI3K/Akt/ mTOR), rat sarcoma/ extracellular regulated protein kinases/MYC (RAS/ERK/MYC) can be inhibited [10]. Thus, only patients with EGFR activation mutations can benefit from this mechanism. Therefore, EGFR-TKIs are regarded as an important breakthrough in the targeted therapies in NSCLC [11]. Current studies indicate that many miRNAs are widely involved in EGFR-TKIs therapies and the expressions of miRNAs are different between the resistant and sensitive tumor cells under the EGFR-TKIs therapy [2]. For example, miRNA-200c targets Zinc finger E-box binding homeobox1 (ZEB1) protein or inhibits PI3K/Akt signaling pathway to

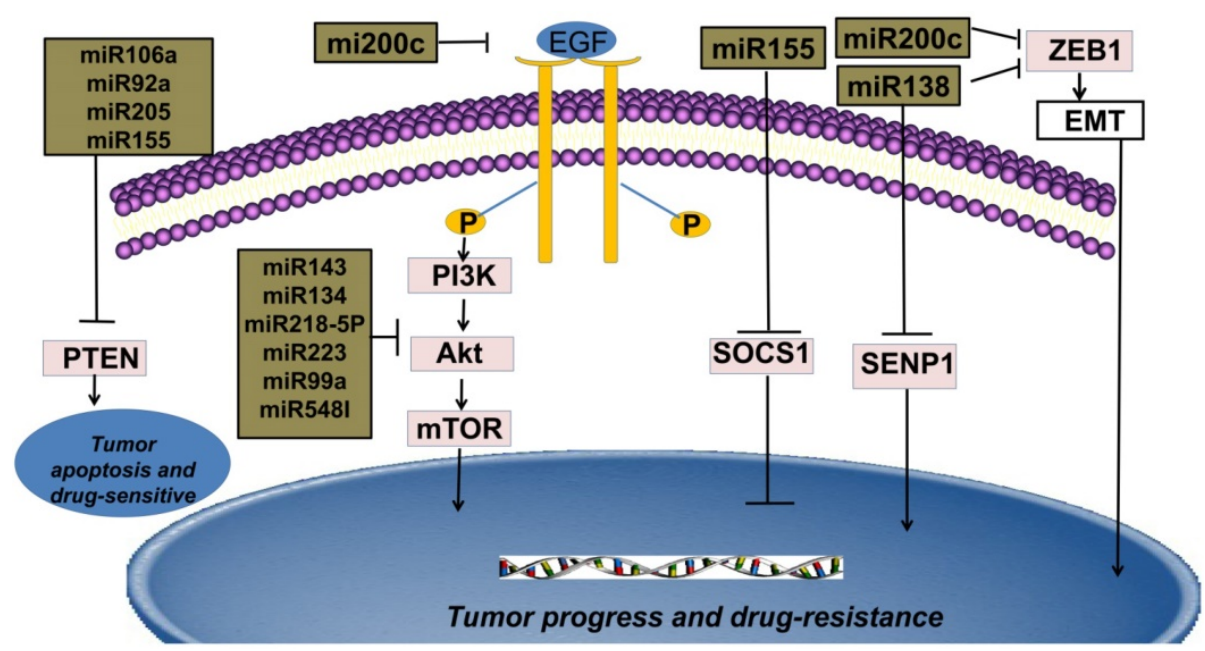

Figure 1. MicroRNAs and their targets involve in the drug-response in NSCLC. enhance gefitinib sensitivity [12]. MiRNA-126 induces autophagy by suppressing insulin receptor substrate-1 (IRS-1) and PI3K/AKT signaling pathway in many tumors including colon cancer, NSCLC and malignant mesothelioma [13]. Likewise, miRNA-143, miRNA-134, miRNA-218-5p can directly bind 3'UTR of EGFR and suppress migration and proliferation of tumor cells $[14,15,16]$. It is believed that miRNA-223, miRNA-99a and miRNA-548I target insulin-like growth factors 1 receptor/phosphoinositide-3 kinase/ protein kinase-B/mammalian target of rapamycin (IGF1R/PI3K/Akt/mTOR) signaling pathway and can inhibit proliferation, migration, and invasion of tumor cell lines. [17, 18, 19]. The interaction of phosphoinositide 3-kinase-seven in absentia homolog 2 (PI3K-SIAH2) regulated by miRNA-30-5p family including miRNA-30a-5p, miRNA-30b-5p, miRNA30c-5p, miRNA-30d-5p, and miRNA-30e-5p, can be considered as a potential treatment target and theranostic panel in NSCLC, particularly adenocarcinoma [20]. All above these anti-oncogenic miRNAs have the potential to enhance tumor sensitivity toward targeted therapies.

\section{MiRNAs and sensitivity to radio-chemotherapy of NSCLC}

MiRNAs contribute extensively to platinumbased chemotherapy in NSCLC. For example, by targeting polycystic kidney disease-2 (PKD2), miRNA-106b-5p increases chemosensitivity in NSCLC [21]. By binding to the high mobility group A 2(HMGA2)-mediated E2F1-Akt pathway, miRNA-26a retards cell growth and sensitizes cell response to cisplatin (CDDP) [22]. The miRNA 17 family can suppress TGF $\beta$ R2, a central receptor of the tumor growth factor- $\beta$ (TGF $\beta$ ) signal pathway, and reverse cisplatin-resistance in NSCLC [23]. It is observed that frizzled class receptor1 (FZD1), involve in tumor proliferation and chemoresistance, and is also a target gene of miRNA-135b [24]. In the therapies of cis-diaminedichloroplatinum

(CDDP), miRNA-379 functions as a therapeutic enhancer by binding to eukaryotic initiation factor 4G2 (EIF4G2) in NSCLC [25]. According to emerging data, miRNA-296-3p attenuates cell proliferation and increases cell sensitivity to 
5-FU, DDP and paclitaxel by targeting C-X3-C motif chemokine receptor1 (CX3CR1) in NSCLC [26]. Also, knockdown of cyclin J (CCNJ) can increase the sensitivity of DDP-based chemotherapy and the effect can be achieved by miRNA-146a [27]. Moreover, miRNA-9600, a novel molecule, is identified to impair the expression of signal transducer and activator of transcription 3 (STAT3) and thus inhibits cell growth and enhances paclitaxel sensitivity in NSCLC [28]. Furthermore, up-regulation of miRNA-589 and miRNA-1244 inhibit viability of tumor cells and reverse resistance to cisplatin in NSCLC [29]. Phosphatidylethanolamine-

binding protein 4 (PEBP4) is a crucial tumor promoter in NSCLC cell lines, which is downregulated by miRNA-34a [30]. Interestingly, miRNA-181b inhibits chemoresistance and promotes chemosensitivity by binding TGF $\beta$ R1/Smad signal pathway [31]. Over-expression of miRNA-7 sensitizes NSCLC cells to paclitaxel and promotes cell apoptosis [32].

Similar to surgery, radiations are deemed to minimize and shrink the volume of tumors in terms of local control, an irreplaceable therapeutic choice for patients with NSCLC [33]. Likewise, some miRNAs are closely involved in the radiotherapy process. MiRNA-29c enhances radiosensitivity in NSCLC by targeting BCL2 and MCL1, both of which are anti-apoptotic genes [34]. Also, miRNA-200c enhances radiosensitivity in NSCLC by retarding oxidative response genes and hampering DNA repair [35]. Experimental results establish that miRNA-328-3p increases radiosensitivity by inducing DNA double strand breaks (DSBs) [36]. In addition, miRNA-138 promotes radiosensitivity and leads to apoptosis by targeting Sentrin/SUMO-specific protease 1 (SENP1), which is a marker of radioresistance in NSCLC [37]. Overexpression of miRNA-449a accelerates apoptosis in NSCLC tissue under radiotherapy [38]. Used as a tumor suppressor in patients with NSCLC, miRNA451 can reverse both chemotherapy and radiotherapy resistance by targeting c-Myc [39]. MiRNA-184 inhibits NSCLC cells survival and invasion capability by binding c-Myc and Bcl-2, also promote cisplatin response in NSCLC cells [40]. CDKN1A (cyclindependent kinase inhibitor 1A) can arrest cells at G1 phase and RAD21 (Rad21 homolog (S. pombe)) and repair DNA damage caused by cisplatin, which are two crucial factors to platinum-based chemotherapy resistance [41]. Inspiring, miRNA-17 family and miRNA-92 family can reverse cisplatin resistance by targeting CDKN1A and RAD21 [41]. Let-7 serves as a tumor suppressor by reducing the expression of oncogenic protein LIN28, and reverse drug-resistance both in radiotherapy and chemotherapy [42].

\section{MiRNAs and resistance to therapy of NSCLC}

\section{MiRNAs and resistance to targeted therapy of NSCLC}

Multiple studies reveal that the secondary T790 mutation event is related to EGFR-TKIs resistance, which account for about $50 \%$ of acquired resistance $[43,44]$. These mutations influence the adenosine triphosphate (ATP)-binding region of EGFR, which is the same region where TKIs bind [45]. The activation of downstream signal pathways of EGFR is also related to the loss of its negative regulatorphosphatase and tensin homology deleted on chromosome ten (PTEN) [46]. The concrete mechanisms of drug resistance are still unknown, but it is established that the expression levels of miRNAs are different in NSCLC cell lines compared with adjacent normal tissues. For instance, miRNA-21, one of the most familiar miRNA, is in 3'UTR of the vacuole membrane protein 1 gene at chromosome 17q23.1, the easiest amplified region in vivo [47]. MiRNA-21 promotes proliferation of NSCLC by inhibiting the expression of PTEN, a unique tumor suppressor gene in vivo, and induces EGFR-TKIs resistance in NSCLC [48]. In addition, miRNA-106a, miRNA-92a and miRNA-205 can also play the similar effects by binding to PTEN $[49,50,51]$. MiRNA-155 acts as an important oncogenic gene in vivo, which targets the suppressor of cytokine signaling 1 (SOCS1) gene, activates Akt signaling pathway and inhibits tumor suppressors including PTEN [52, 53]. Meanwhile, data from Kitamura et al. suggested that miR-134/ $487 \mathrm{~b} / 655$ cluster contributed to the TGF- $\beta 1$-induced EMT phenomenon and affected the resistance to gefitinib by directly targeting MAGI2, in which suppression subsequently caused loss of PTEN stability in NSCLC cells [54].

\section{MiRNAs and resistance to radio-chemotherapy of NSCLC}

Besides targeted therapy strategy, miRNAs are also extensively involved in the various mechanisms of radio-chemotherapy of NSCLC. Hypoxia-inducible factor- $1 \alpha$ (HIF $1 \alpha$ ), an upstream signaling molecule of glycolysis, can be activated under hypoxia and upregulated in radiation-resistant cell lines [55]. MiRNA-21 leads to radioresistance in NSCLC by upregulating HIF1 $\alpha$-induced glycolysis [55]. Besides HIF1, miRNA-210 also repair genomic double-strand breaks (DSBs) induced by radiation, and result in the radioresistance of NSCLC cells [56]. MiRNA-25, a crucial tumor suppressor gene, reduce sensitivity to radiation by binding the $\mathrm{B}$-cell translocation gene 2 (BTG2) in NSCLC [57]. The radioprotector, miRNA- 
1323, can weaken radiosensitivity by promoting the expression of protein kinase, DNA-activated, catalytic polypeptide (PRKDC) in NSCLC [58].

In addition, miRNAs also regulate the process of chemotherapy besides targeted therapy and radiotherapy. Wnt/ $\beta$-catenin signaling pathway has been reported to be a crucial mechanism of proliferation, growth, and drug resistance of tumors including NSCLC [59]. Interestingly, miR-181c leads to cisplatin resistance in NSCLC cells by targeting Wnt inhibition factor 1 [59]. Similarly, Li et al. showed that inhibition of miR-196a could reverse cisplatin resistance of A549/DDP cell lines [60]. The role and mechanism of miR-196a might relate with inhibition of drug efflux, down-regulation of drug-resistant protein expression, cell apoptosis, and cell proliferation suppression [60]. MiRNA-21 suppresses drosophila mothers against decapentaplegic protein7 (SMAD7), a key inhibitor of TGF $\beta$ receptor signaling and thus reduces carboplatin-response in NSCLC cells [61].

\section{MiRNAs and sensitivity to therapy of NSCLC by preventing EMT}

Among targeted therapies, conventional chemotherapy and radiotherapy, anti-oncogenes extensively affect therapy sensitivity. Consequently, upregulation of these genes is closely related to tumor regression and apoptosis in NSCLC. Contrarily, lower expression of these anti-oncogenes might induce invasion and drug resistance of tumor cells. Meanwhile, several tumor-promoting miRNAs have shown an ability to enhance the proliferation and invasion of NSCLC cells.

Epithelial-Mesenchymal transition (EMT) is an important step for cancer metastasis. Both epithelial and mesenchymal molecules are crucial factors for cell adhesion, cell polarity and cell shape. While upregulation of E-cadherin is closely linked to lower grade tumor, rising mesenchymal is related to invasive tumor type in vivo or vitro $[62,63]$. Recent studies have shown that Snail and ZEB1 are two significant EMT promoters in NSCLC [64]. MiRNA-218 binds to Slug/ZEB2 signaling pathway, one of the EMT-related factors in various solid tumors, and thus improves both anti-cancer ability and therapeutic efficacy in NSCLC [65]. Likewise, miRNA-193a and miRNA-132 can prevent TGF- $\beta 1$-induced EMT thus improve both anti-cancer ability and therapeutic efficacy in NSCLC $[66,67]$. Moreover, miRNA-200c is shown to act as an anti-oncogene by targeting ZEB-induced EMT or PI3K/Akt signaling pathway and overcome gefitinibresistance in NSCLC $[12,35,68]$. MiRNA-138 can deter chemo-resistance by inhibiting ZEB-induced EMT in patients with NSCLC [69]. Likewise, by binding to ROCK2, an important promoter of EMT, miRNA-101 might gain advanced patients with a longer disease-free survival (DFS) [70]. MiRNA-338$3 p$ can inhibit the growth, migration and invasion of NSCLC by controlling Sox4-induced EMT [71]. Upregulations of these miRNAs can enhance therapy-sensitivity and thus benefit patients.

\section{MiRNAs and resistance to therapy of NSCLC by inducing EMT}

EMT acts as an important element for tumor proliferation and metastasis in NSCLC. The activation of EMT is correlated with poor prognosis for patients accepting either conventional radio-chemotherapies or novel targeted therapies, which indicates the roles of EMT in drug resistance of human cancers [72, 73, 74, 75]. EMT, the most researched mechanism in tumor development, indicates a more aggressive tumor class, higher migration rate and extreme drug-resistance [73, 76]. Instead of an independent biological process, EMT is also responsible for EGFR-TKIs resistance by activating the PI3K/Akt signal pathway [77]. Interestingly, miRNA-221/222 and miRNA-30b-c are upregulated after EMT amplification, which also serve as downstream gene of EGFR [78]. According to analyses on expression of miRNAs between tumor tissues and adjacent normal tissues, there are direct relationships between EMT and oncogenic miRNAs, such as miRNA-221/222 and miRNA-30b-c, indicate for lower drug response, which argues that downregulation of these miRNA genes might be a promising direction for more effective therapies [78].

MiRNAs inhibit tumor developmental processes through multiple mechanisms, which indicate that monoclonal antibody, combined with EGFR-TKIs and chemotherapy become an efficient comprehensive therapeutic strategy for patients with NSCLC [79]. For instance, miRNA-92a triggers EMT by activating PTEN/PI3K/Akt signaling pathway in NSCLC [80]. One novel oncogene, miRNA-181b-5p regulates TGF- $\beta 1$-induced EMT in NSCLC [81]. Overall, upregulation of these oncogenes is directly involved in proliferation, invasion and drug resistance. Meanwhile, these categories of miRNAs are biomarkers for poor prognosis and lower DFS rate of patients with NSCLC.

\section{MiRNAs themselves were modulated in therapy of NSCLC}

Alterations of miRNAs expression after radiotherapy, chemotherapy or targeted therapy are correlated with therapy response. According to data from published literatures, miRNAs and multiple 
treatment plans mutually affect in the clinical processes [82]. In the therapies of NSCLC, miRNAs are observed to have dynamic changes. For instance, after EGFR signal pathway was activated, there was elevated miRNA-21 in NSCLC cell lines [83]. Recent studies show that the expression of miRNA-30b-c/ 221/222 are downregulated in EGFR-TKIs responsive cell lines, with upregulation of Bcl-2 interacting mediator of cell death (BIM) and apoptotic peptidase activating factor1 (APAF-1) [78]. MiRNA-26a, a tumor suppressor molecule, downregulate in CDDPresistant sample [22]. Oncogenes, such as miRNA-155, are downregulated in NSCLC cells with gefitinib therapy [52, 53]. These oncogenic miRNAs might upregulate in the resistant cell lines of NSCLC and have the potential to weaken therapeutic efficacy. Conversely, tumor suppressors can also be upregulated in sensitive NSCLC cell lines and adjust therapeutic response. Some miRNAs target EGFR pathway, such as miRNA-143, miRNA-134 and miRNA-218-5p, which are directly related to EGFR-TKIs response in patients with NSCLC $[14,15$, 16]. These miRNAs can reverse drug resistance in the clinical therapy process, which might be a novel molecular candidate for targeted therapy in the future.

\section{MiRNAs as biomarkers for therapy in NSCLC}

Most NSCLC patients are diagnosed at advanced stage and therefore have an unfavorable clinical prognosis. Due to the side effects of chemotherapy and radiation treatment for NSCLC patients, there is an urgent need on personalized targeted medicine. With high sensitivity and accuracy, dysregulated expression levels of miRNA in vivo may indicate the occurrence of NSCLC earlier than the appearance of

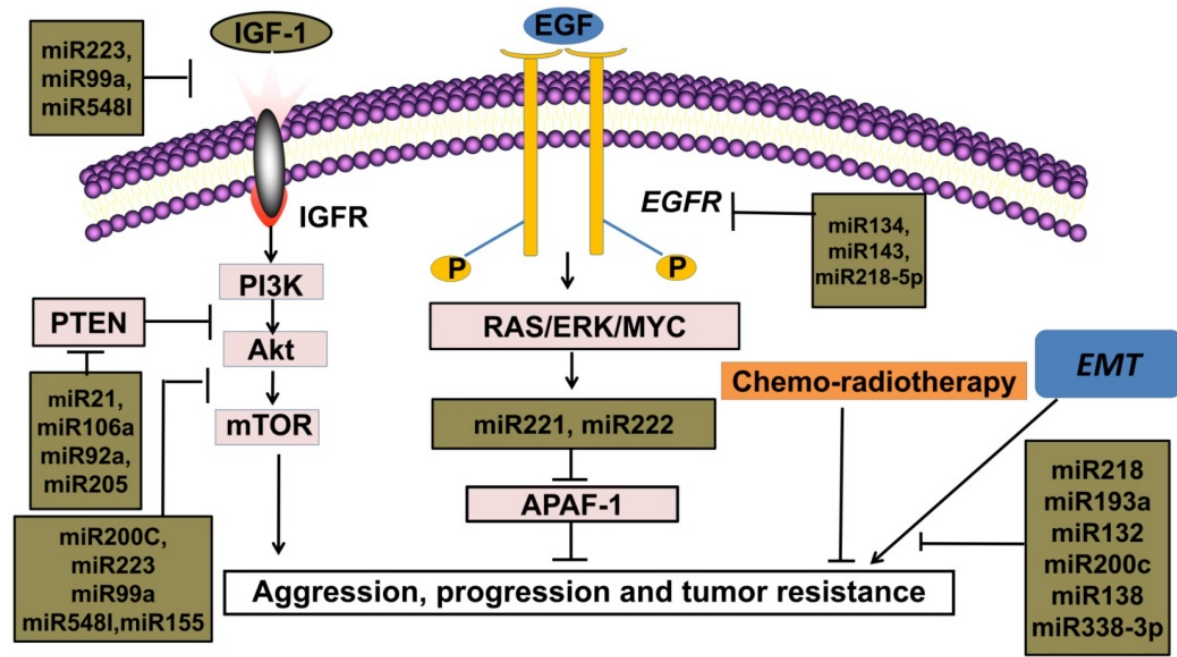

Figure 2. MicroRNAs participate in modulation of therapies-effect such as novel targeted therapy, chemotherapy and radiotherapy. symptoms and abnormal CT images [1]. Regulating expression of targeted genes in post-transcription period, miRNAs involve in biological signal channels and thus influence therapeutic effects. This feature indicates that miRNAs will be applied as powerful targets to effective therapy strategy $[4,5]$. The most promising example is that NSCLC cell lines with EGFR mutation are more sensitive to EGFR-TKIs than those wild types, and mutation events might relate with expression level of these miRNAs [11]. The EMT promotor, miRNAs, can be detected in vivo, which indicate high heterogeneous property. It is demonstrated that approximately half of miRNAs are located in sensitive regions of chromosomes, with the high possibility of amplification or deletion [84]. These unstable miRNAs play a crucial role in determining effects of therapies and differential expressions of these miRNAs between drug-sensitive and drug-resistance in the NSCLC cell lines have been shown in previous studies. Therefore, the expression level of miRNAs can predict effects and even guide therapies, including radiotherapy, chemotherapy and targeted therapies. More research data show that abnormal expression of miRNAs play important roles in the progression and drug-resistance of NSCLC by modulating oncogenic or tumor-suppressing genes and can act as biomarkers for therapy in NSCLC. These miRNAs with high sensitivity and specificity can serve as biomarkers in targeted therapies especially for advanced patients with NSCLC.

\section{Conclusions and future directions}

Different kinds of miRNAs play different roles by binding to specific targets. These studies revealed that anti-oncogenes miRNAs reverse EGFR-TKIs resistance in the NSCLC by relevant targets (Table 1). Meanwhile, miRNAs are involved in almost every step of the tumor progression (Table 2 and Figure 2). Although published literature indicated a correlation between miRNAs and the therapy of NSCLC, the detailed mechanisms involved have not been clarified, such as the relationship between secondary T790 mutation and miRNAs. Further research is needed to better understand how to overcome drug resistance completely. In summary, miRNAs may serve as powerful biomarkers for diagnosis or prognosis and will be novel targeting therapy in NSCLC. 
Table 1. Anti-oncogenetic miRNAs and target genes relate to drug-sensitivity

\begin{tabular}{|c|c|c|}
\hline MiRNAs & Related Mechanisms & References \\
\hline miRNA-126 & Targets IRS1, EGFR & [13] \\
\hline miRNA-143 & Targets EGFR & {$[14]$} \\
\hline miRNA-134 & Targets EGFR & {$[15]$} \\
\hline miRNA-218-5p & Targets EGFR & [16] \\
\hline miRNA-223 & Targets IGF1R/PI3K/Akt/mTOR & [17] \\
\hline miRNA-99a & Targets IGF1R/PI3K/Akt/mTOR & [18] \\
\hline miRNA-5481 & Targets IGF1R/PI3K/Akt/mTOR & [19] \\
\hline miRNA-30-5p & Targets PI3K-SIAH2 & [20] \\
\hline miRNA-106b-5p & Targets PKD2 & {$[21]$} \\
\hline miRNA-26a & Targets Akt & {$[22]$} \\
\hline miRNA-17 & Suppresses TGF $\beta 2$ & [23] \\
\hline miRNA-135b & Targets FZD1 & {$[24]$} \\
\hline miRNA-379 & Targets EIF4G2 & [25] \\
\hline miRNA-296-3p & Targets CX3CR1 & [26] \\
\hline miRNA-146a & Inhibits CCNJ & [27] \\
\hline miRNA-9600 & Inhibits STAT3 & [28] \\
\hline miRNA-34a & Inhibits PEBP4 & [30] \\
\hline miRNA-181b & Binds to TGF $\beta$ R1/smad & [31] \\
\hline miRNA-451 & Targets c-Myc & [39] \\
\hline miRNA-184 & Binds to c-Myc and Bcl-2 & {$[40]$} \\
\hline miRNA-17 family & Targets CDKN1A and RAD21 & {$[41]$} \\
\hline miRNA-19 family & Targets CDKN1A and RAD21 & {$[41]$} \\
\hline Let-7 & Inhibits LIN28 & {$[42]$} \\
\hline miRNA-218 & Inhibits Slug/ZEB2-induced EMT & [65] \\
\hline miRNA-193a,miR-132 & Prevent TGF- $\beta$ 1-induced EMT & {$[66,67]$} \\
\hline miRNA-200c & $\begin{array}{l}\text { Targets ZEB-induced EMT and } \\
\text { PI3K/AKt }\end{array}$ & {$[12,35,68]$} \\
\hline miRNA-138 & Inhibits ZEB-induced EMT and SENP1 & {$[37,69]$} \\
\hline miRNA-101 & Binds to ROCK2 & {$[70]$} \\
\hline miRNA-338-3p & Controls SOX4-induced EMT & [71] \\
\hline \multicolumn{3}{|c|}{$\begin{array}{l}\text { Note: MiRNAs are involved in therapeutic processes by binding to target gene and } \\
\text { sensitize drug-response. }\end{array}$} \\
\hline \multicolumn{3}{|c|}{$\begin{array}{l}\text { miRNA: microRNA; IRS-1: insulin receptor substrate-1; EGFR: Epidermal growth } \\
\text { factor receptor; IGF1R/PI3K/Akt/mTOR: insulin-like growth factors } 1 \text { receptor/ } \\
\text { phosphoinositide-3 kinase/protein kinase-B/mammalian target of rapamycin; } \\
\text { PI3K-SIAH2: Phosphoinositide 3-kinase-seven in absentia homolog2; PKD2: } \\
\text { polycystic kidney disease-2; TGF } \beta 2 \text { : transforming growth factor } \beta 2 \text {; FZD1: frizzled } \\
\text { class receptor 1; EIF4G2: eukaryotic initiation factor 4G2; CX3CR1: C-X3-C motif } \\
\text { chemokine receptor1; CCNJ: cyclin J; STAT3: Signal transducers and activators of } \\
\text { transcription 3; PEBP4: phosphatidylethanolamine-binding protein 4; CDKN1A: } \\
\text { cyclin-dependent kinase inhibitor 1A; RAD21: Rad21 homolog (S. pombe); ZEB1: } \\
\text { Zinc finger E-box binding homeobox1; EMT: Epithelial-Mesenchymal Transition; } \\
\text { SENP1: Sentrin/SUMO-specific protease 1; SOX4: SRY-box4. }\end{array}$} \\
\hline
\end{tabular}

Table 2. Oncogenetic miRNAs and target genes relate to drug-resistance

\begin{tabular}{|c|c|c|}
\hline MiRNAs & Related Mechanisms & References \\
\hline miRNA-106a & Inhibits PTEN & [49] \\
\hline miRNA-92a & Inhibits PTEN & {$[50]$} \\
\hline miRNA-205 & Inhibits PTEN & [51] \\
\hline miRNA-155 & $\begin{array}{l}\text { Inhibits caspase-3, SOCS1 and PTEN, activates } \\
\text { AKT, }\end{array}$ & {$[52,53]$} \\
\hline miRNA-210 & Upregulates HIF $1 \alpha$ & [56] \\
\hline miRNA-25 & Inhibits BTG2 & [57] \\
\hline miRNA-1323 & Promotes PRKDC & {$[58]$} \\
\hline miRNA-181c & Targets Wnt inhibition factor 1 & [59] \\
\hline miRNA-21 & Inhibits PTEN and SMAD7, promotes HIF $1 \alpha$ & {$[47,55,61]$} \\
\hline \multicolumn{3}{|c|}{$\begin{array}{l}\text { Note: MiRNAs are involved in therapeutic processes by binding to target gene and } \\
\text { hamper drug-response. }\end{array}$} \\
\hline \multicolumn{3}{|c|}{$\begin{array}{l}\text { miRNA: microRNA; PTEN: phosphatase and tensin homology deleted on } \\
\text { chromosome ten; SOCS1: Suppressor of Cytokine Signaling 1; Akt: protein kinase-B; HIF } \\
\text { 1 } \alpha \text { : Hypoxia-inducible factor- } 1 \alpha \text {; BTG2: B-cell translocation gene 2; PRKDC: protein } \\
\text { kinase, DNA-activated, catalytic polypeptide; SMAD7: drosophila mothers against } \\
\text { decapentaplegic protein7. }\end{array}$} \\
\hline
\end{tabular}

\section{Abbreviations}

NSCLC: non-small cell lung cancer; miRNAs: microRNAs; ORFs: open reading frames; pri-miRNA: primary microRNA; pre-miRNA: precursor microRNA; RISC: RNA induced silencing complex; EGFR-TK: epidermal growth factor receptor-tyrosine kinase; PI3K: phosphoinositide-3 kinase; Akt: protein kinase-B; mTOR: mammalian target of rapamycin; RAS: rat sarcoma; ERK: extracellular regulated protein kinases; ZEB1: Zinc finger E-box binding homeobox1; IRS1: insulin receptor substrate-1; IGF1R: insulin-like growth factor 1 receptor; PI3K-SIAH2: phosphoinositide 3-kinase-seven in absentia homolog 2; PKD2: polycystic kidney disease-2; HMGA2: high mobility group A 2; CDDP: cisplatin, cis-diaminedichloroplatinum; TGF $\beta$ : tumor growth factor- $\beta$; FZD1: frizzled class receptor1; EIF4G2: eukaryotic initiation factor 4G2; PKD2: polycystic kidney disease-2; CX3CR1: C-X3-C motif chemokine receptor1; CCNJ: cyclin J; PEBP4: Phosphatidylethanolamine-binding protein 4; DSBs: DNA double strand breaks; STAT3: signal transducer and activator of transcription 3; PEBP4: Phosphatidylethanolaminebinding protein 4; SENP1: Sentrin/SUMO-specific protease 1; CDKN1A: cyclin-dependent kinase inhibitor 1A; RAD21: Rad21 homolog (S. pombe); PTEN: phosphatase and tensin homology deleted on chromosome ten; SOCS1: suppressor of cytokine signaling 1; HIF $1 \alpha$ : Hypoxia-inducible factor- $1 \alpha$; BTG2: B-cell translocation gene 2; PRKDC: protein kinase, DNA-activated, catalytic polypeptide; SMAD7: drosophila mothers against decapentaplegic protein7; EMT: Epithelial-Mesenchymal transition; DFS: disease-free survival; BIM: Bcl-2 interacting mediator of cell death; APAF-1: apoptotic peptidase activating factor1.

\section{Acknowledgments}

This work was supported by the National Natural Science Foundations of China (No: 81773218; 81472773 and 81703009). Thank Christopher Qoyawayma, who works in the Pediatric division of the Children's Hospital of Pittsburgh of UPMC, University of Pittsburgh, Pittsburgh, Pennsylvania, United States; for his help with the English-language editing of the manuscript.

\section{Competing Interests}

The authors have declared that no competing interest exists.

\section{References}

1. Hou J, Meng F, Chan LW, Cho WC, Wong SC. Circulating Plasma MicroRNAs As Diagnostic Markers for NSCLC. Front Genet. 2016; 7: 193-216. 
2. Mizuno $\mathrm{K}$, Mataki $\mathrm{H}$, Seki $\mathrm{N}$, Kumamoto $\mathrm{T}$, Kamikawaji $\mathrm{K}$, Inoue $\mathrm{H}$. MicroRNAs in non-small cell lung cancer and idiopathic pulmonary fibrosis. J Hum Genet. 2017; 62(1): 57-65.

3. Davies J, Patel M, Gridelli C, de Marinis F, Waterkamp D, McCusker ME. Real-world treatment patterns for patients receiving second-line and third-line treatment for advanced non-small cell lung cancer: A systematic review of recently published studies. PLoS One. 2017; 12(4).

4. Markou A, Sourvinou I, Vorkas PA, Yousef GM, Lianidou E. Clinical evaluation of microRNA expression profiling in non-small cell lung cancer. Lung Cancer. 2013; 81(3): 388-396.

5. Price C, Chen J. MicroRNAs in Cancer Biology and Therapy: Current Status and Perspectives. Genes Dis.2014; 1(1): 53-63.

6. Gan TQ, Xie ZC, Tang RX, Zhang TT, Li DY, Li ZY, et al. Clinical value of miR-145-5p in NSCLC and potential molecular mechanism exploration: A retrospective study based on GEO, qRT-PCR, and TCGA data. Tumour Biol. 2017; 39(3):1010428317691683.

7. Sin TK, Wang F, Meng F, Wong SC, Cho WC, Siu PM, et al. Implications of MicroRNAs in the Treatment of Gefitinib-Resistant Non-Small Cell Lung Cancer. Int J Mol Sci. 2016; 17(2):237.

8. Zang H, Wang W, Fan S. The role of microRNAs in resistance to targeted treatments of non-small cell lung cancer. Cancer Chemother Pharmacol. 2017; 79(2): 227-231

9. Zhan Y, Zang H, Feng J, Lu J, Chen L, Fan S. Long non-coding RNAs associated with non-small cell lung cancer. Oncotarget. 2017; 8(40):69174-69184

10. Wang X, Goldstein D, Crowe PJ, Yang JL. Next-generation EGFR/HER tyrosine kinase inhibitors for the treatment of patients with non-small-cell lung cancer harboring EGFR mutations: a review of the evidence. Onco Targets Ther. 2016; 9: 5461-5473.

11. Que D, Xiao H, Zhao B, Zhang X, Wang Q, Xiao H, et al. EGFR mutation status in plasma and tumor tissues in non-small cell lung cancer serves as a predictor of response to EGFR-TKI treatment. Cancer Biol Ther. 2016; 17(3): 320-327.

12. Zhou G, Zhang F, Guo Y, Huang J, Xie Y, Yue S, et al. MiR-200c enhances sensitivity of drug-resistant non-small cell lung cancer to gefitinib by suppression of PI3K/Akt signaling pathway and inhibites cell migration via targeting ZEB1. Biomed Pharmacother. 2017; 85: 113-119.

13. Tomasetti M, Monaco F, Manzella N, Rohlena J, Rohlenova K, Staffolani S, et al. MicroRNA-126 induces autophagy by altering cell metabolism in malignant mesothelioma. Oncotarget. 2016; 7(24): 36338-36352.

14. Zhang HB, Sun LC, Ling L, Cong LH, Lian R. MiR-143 suppresses the proliferation of NSCLC cells by inhibiting the epidermal growth factor receptor. Exp Ther Med. 2016; 12(3): 1795-1802.

15. Qin Q, Wei F, Zhang J, Wang X, Li B. MiR-134 inhibits non-small cell lung cancer growth by targeting the epidermal growth factor receptor. J Cell Mol Med. 2016; 20(10): 1974-1983.

16. Zhu K, Ding H, Wang W, Liao Z, Fu Z, Hong Y, et al. Tumor-suppressive miR-218-5p inhibits cancer cell proliferation and migration via EGFR in non-small cell lung cancer. Oncotarget. 2016; 7(19): 28075-28085.

17. Han J, Zhao F, Zhang J, Zhu H, Ma H, Li X, et al. MiR-223 reverses the resistance of EGFR-TKIs through IGF1R/PI3K/Akt signaling pathway. Int J Oncol. 2016; 48(5): 1855-1867.

18. Yu SH, Zhang CL, Dong FS, Zhang YM. MiR-99a suppresses the metastasis of human non-small cell lung cancer cells by targeting AKT1 signaling pathway. J Cell Biochem. 2015; 116(2): 268-276

19. Liu C, Yang H, Xu Z, Li D, Zhou M, Xiao K, et al. MicroRNA-548l is involved in the migration and invasion of non-small cell lung cancer by targeting the AKT1 signaling pathway. J Cancer Res Clin Oncol. 2015; 141(3): 431-441.

20. Chan LW, Wang F, Meng F, Wang L, Wong SC, Au JS, et al. MiR-30 Family Potentially Targeting PI3K-SIAH2 Predicted Interaction Network Represents a Novel Putative Theranostic Panel in Non-small Cell Lung Cancer. Front Genet. 2017; 8: 8

21. Yu S, Qin X, Chen T, Zhou L, Xu X, Feng J. MicroRNA-106b-5p regulates cisplatin chemosensitivity by targeting polycystic kidney disease-2 in non-small-cell lung cancer. Anticancer Drugs. 2017 28(8):852-860.

22. Zang H, Peng J, Wang W, Fan S. Roles of microRNAs in the resistance to platinum based chemotherapy in the non-small cell lung cancer. J Cancer. 2017; 8(18):3856-3861.

23. Jiang Z, Yin J, Fu W, Mo Y, Pan Y, Dai L, et al. MiRNA 17 family regulates cisplatin-resistant and metastasis by targeting TGFbetaR2 in NSCLC. PLoS One. 2014; 9(4):e94639

24. Su W, Mo Y, Wu F, Guo K, Li J, Luo Y, et al. MiR-135b reverses chemoresistance of non-small cell lung cancer cells by downregulation of FZD1. Biomed Pharmacother. 2016; 84:123-129.

25. Hao GJ, Hao HJ, Ding YH, Wen H, Li XF, Wang QR, et al. Suppression of EIF4G2 by miR-379 potentiates the cisplatin chemosensitivity in no small cell lung cancer cells. FEBS Lett. 2017; 591(4): 636-645.

26. Luo W, Lin Y, Meng S, Guo Y, Zhang J, Zhang W. MiRNA-296-3p modulates chemosensitivity of lung cancer cells by targeting CX3CR1. Am J Transl Res. 2016; 8(4): 1848-1856.

27. Shi $\mathrm{L}, \mathrm{Xu} \mathrm{Z,Wu} \mathrm{G}$, Chen X, Huang Y, Wang Y, et al. Up-regulation of miR-146a increases the sensitivity of non-small cell lung cancer to DDP by downregulating cyclin J. BMC Cancer. 2017; 17(1): 138.

28. Sun CC, Li SJ, Zhang F, Zhang YD, Zuo ZY, Xi YY, et al. The Novel miR-9600 Suppresses Tumor Progression and Promotes Paclitaxel Sensitivity in
Non-small-cell Lung Cancer Through Altering STAT3 Expression. Mol Ther Nucleic Acids. 2016; 5(11):e387

29. Li W, Wang W, Ding M, Zheng X, Ma S, Wang X. MiR-1244 sensitizes the resistance of non-small cell lung cancer A549 cell to cisplatin. Cancer Cell Int. 2016; 16: 30

30. Yu G, Zhong N, Chen G, Huang B, Wu S. Downregulation of PEBP4, a target of miR-34a, sensitizes drug-resistant lung cancer cells. Tumour Biol. 2014; 35(10):10341-10349.

31. Wang X, Chen X, Meng Q, Jing H, Lu H, Yang Y, et al. MiR-181b regulates cisplatin chemosensitivity and metastasis by targeting TGF $\beta R 1 / S m a d$ sionaling pathway in NSCLC. Sci Rep. 2015; 5:17618.

32. Liu R, Liu X, Zheng $Y$, Gu J, Xiong S, Jiang P, et al. MicroRNA-7 sensitizes non-small cell lung cancer cells to paclitaxel. Oncol Lett. 2014; 8(5): 2193-2200.

33. Alongi F, Arcangeli S, De Bari B, Giaj-Levra N, Fiorentino A, Mazzola R, et al. Stage-I small cell lung cancer: A new potential option for stereotactic ablative radiation therapy? A review of literature. Crit Rev Oncol Hematol. 2017; 112: 67-71.

34. Arechaga-Ocampo E, Lopez-Camarillo C, Villegas-Sepulveda N, Gonzalez-De la Rosa CH, Perez-Añorve IX, Roldan-Perez R, et al. Tumor suppressor miR-29c regulates radioresistance in lung cancer cells. Tumour Biol. 2017; 39(3):1010428317695010.

35. Cortez MA, Valdecanas D, Zhang X, Zhan Y, Bhardwaj V, Calin GA, et al. Therapeutic delivery of miR-200c enhances radiosensitivity in lung cancer. Mol Ther. 2014; 22(8):1494-1503.

36. Ma W, Ma CN, Zhou NN, Li XD, Zhang YJ. Up-regulation of miR-328-3p sensitizes non-small cell lung cancer to radiotherapy. Sci Rep. 2016; 6:31651.

37. Yang $\mathrm{H}$, Tang $\mathrm{Y}$, Guo $\mathrm{W}$, Du Y, Wang Y, Li P, et al. Up-regulation of microRNA-138 induce radiosensitization in lung cancer cells. Tumour Biol. 2014; 35(7): 6557-6565

38. Liu YJ, Lin YF, Chen YF, Luo EC, Sher YP, Tsai MH, et al. MicroRNA-449a enhances radiosensitivity in CL1-0 lung adenocarcinoma cells. PLoS One. 2013; 8(4):e62383.

39. Wang R, Chen DQ, Huang JY, Zhang K, Feng B, Pan BZ, et al. Acquisition of radioresistance in docetaxel-resistant human lung adenocarcinoma cells is linked with dysregulation of miR-451/c-Myc-survivin/rad-51 signaling. Oncotarget. 2014; 5(15): 6113-6129.

40. Tung MC, Lin PL, Cheng YW, Wu DW, Yeh SD, Chen CY, Lee H. Reduction of microRNA-184 by E6 oncoprotein confers cisplatin resistance in lung cancer via increasing Bcl-2. Oncotarget. 2016; 7(22):32362-32374.

41. Zhao J, Fu W, Liao H, Dai L, Jiang Z, Pan Y, et al. The regulatory and predictive functions of miR-17 and miR-92 families on cisplatin resistance of non-small cell lung cancer. BMC Cancer. 2015; 15: 731.

42. Yin J, Zhao J, Hu W, Yang G, Yu H, Wang R, et al. Disturbance of the let-7/LIN28 double-negative feedback loop is associated with radio- and chemo-resistance in non-small cell lung cancer. PLoS One. 2017; 12(2): 0172787.

43. Yu JY, Yu SF, Wang SH, Bai H, Zhao J, An TT, et al. Clinical outcomes of EGFR-TKI treatment and genetic heterogeneity in lung adenocarcinoma patients with EGFR mutations on exons 19 and 21. Chin J Cancer. 2016; 35: 30.

44. John T, Liu G, Tsao MS. Overview of molecular testing in non-small-cell lung cancer: mutational analysis, gene copy number. protein expression and other biomarkers of EGFR for the prediction of response to tyrosine kinase inhibitors. Oncogene. 2009; 28: S14-23.

45. Pao W, Miller V, Zakowski M, Doherty J, Politi K, Sarkaria I, et al. EGF receptor gene mutations are common in lung cancers from 'never smokers' and are associated with sensitivity of tumors to gefitinib and erlotinib. Proc Natl Acad Sci U S A. 2004; 101(36): 13306-13311.

46. Morgillo F, Della Corte CM, Fasano M, Ciardiello F. Mechanisms of resistance to EGFR-targeted drugs: lung cancer. ESMO Open. 2016; 1(3):e000060.

47. Li X, Zang A, Jia Y, Zhang J, Fan W, Feng J, et al. Triptolide reduces proliferation and enhances apoptosis of human non-small cell lung cancer cells through PTEN by targeting miR-21. Mol Med Rep. 2016; 13(3): 2763-2768.

48. Shen H, Zhu F, Liu J, Xu T, Pei D, Wang R, et al. Alteration in Mir-21/PTEN expression modulates gefitinib resistance in non-small cell lung cancer. PLoS One. 2014; 9(7): e103305.

49. Xie X, Liu HT, Mei J, Ding FB, Xiao HB, Hu FQ, et al. MiR-106a promotes growth and metastasis of non-small cell lung cancer by targeting PTEN. Int J Clin Exp Pathol. 2015; 8(4): 3827-3834.

50. Ren $\mathrm{P}$, Gong $\mathrm{F}$, Zhang $\mathrm{Y}$, Jiang J, Zhang $\mathrm{H}$. MicroRNA-92a promotes growth, metastasis, and chemoresistance in non-small cell lung cancer cells by targeting PTEN. Tumour Biol. 2016; 37(3): 3215-3225.

51. Lei L, Huang Y, Gong W. MiR-205 promotes the growth, metastasis and chemoresistance of NSCLC cells by targeting PTEN. Oncol Rep. 2013; 30(6): 2897-2902.

52. $\mathrm{Xu}$ TP, Zhu $\mathrm{CH}$, Zhang J, Xia R, Wu FL, Han L, et al. MicroRNA-155 Expression has Prognostic Value in Patients with Non-small Cell. Lung Cancer and Digestive System Carcinomas. Asian Pac J Cancer Prev. 2013; 14(12): 7085-7090.

53. Xue $X$, Liu $Y$, Wang $Y$, Meng $M$, Wang $K$, Zang X, et al. MiR-21 and MiR-155 promote non-small cell lung cancer progression by downregulating SOCS1, SOCS6, and PTEN. Oncotarget. 2016; 7(51):84508-8451952.

54. Kitamura K, Seike M, Okano T, Matsuda K, Miyanaga A, Mizutani H, et al. MiR-134/487b/655 cluster regulates TGF- $\beta$-induced epithelial-mesenchymal transition and drug resistance to gefitinib by targeting MAGI2 in lung adenocarcinoma cells. Mol Cancer Ther. 2014; 13(2):444-453. 
55. Jiang $\mathrm{S}$, Wang $\mathrm{R}$, Yan $\mathrm{H}$, Jin $\mathrm{L}$, Dou $\mathrm{X}$, Chen $\mathrm{D}$, MicroRNA-21 modulates radiation resistance through upregulation of hypoxia-inducible factor-1a-promoted glycolysis in non-small cell lung cancer cells. Mol Med Rep. 2016; 13(5):4101-4107.

56. Grosso S, Doyen J, Parks SK, Bertero T, Paye A, Cardinaud B, et al. MiR-210 promotes a hypoxic phenotype and increases radioresistance in human lung cancer cell lines. Cell Death Dis. 2013; 4:e544.

57. He Z, Liu Y, Xiao B, Qian X. MiR-25 modulates NSCLC cell radio-sensitivity through directly inhibiting BTG2 expression. Biochem Biophys Res Commun. 2015; 457(3):235-241

58. Li Y, Han W, Ni TT, Lu L, Huang M, Zhang Y, et al. Knockdown of microRNA-1323 restores sensitivity to radiation by suppression of PRKDC activity in radiation-resistant lung cancer cells. Oncol Rep. 2015; 33(6):2821-2828.

59. Zhang H, Hu B, Wang Z, Zhang F, Wei H Li L. MiR-181c contributes to cisplatin resistance in non-small cell lung cancer cells by targeting Wnt inhibition factor 1. Cancer Chemother Pharmacol. 2017; 80(5):973-984.

60. Li JH, Luo N, Zhong MZ, Xiao ZQ, Wang JX, Yao XY, et al. Inhibition of microRNA-196a might reverse cisplatin resistance of A549/DDP non-small-cell lung cancer cell line. Tumour Biol. 2016; 37(2): 2387-2394.

61. Lin L, Tu HB, Wu L, Liu M, Jiang GN. MicroRNA-21 Regulates Non-Small Cell Lung Cancer Cell Invasion and Chemo-Sensitivity through SMAD7. Cell Physiol Biochem. 2016; 38(6): 2152-2162.

62. Tsoukalas N, Aravantinou-Fatorou E, Tolia M, Giaginis C, Galanopoulos M, Kiakou M, et al. Epithelial-Mesenchymal Transition in Non-Small-cell Lung Cancer. Anticancer Res. 2017; 37(4): 1773-1778.

63. Zhang J, Liang Q, Lei Y, Yao M, Li L, Gao X, et al. SOX4 induces epithelial-mesenchymal transition and contributes to breast cancer progression. Cancer Res. 2012; 72(17): 4597-608.

64. Chao W, Deng JS, Li PY, Liang YC, Huang GJ. 3,4-Dihydroxybenzalactone Suppresses Human Non-Small Cell Lung Carcinoma Cells Metastasis via Suppression of Epithelial to Mesenchymal Transition, ROS-Mediated PI3K/AKT/MAPK/MMP and NFKB Signaling Pathways. Molecules. 2017; 22(4). pii: E537.

65. Shi ZM, Wang L, Shen H, Jiang CF, Ge X, Li DM, et al. Downregulation of miR-218 contributes to epithelial-mesenchymal transition and tumor metastasis in lung cancer by targeting Slug/ZEB2 signaling. Oncogene. 2017; 36(18):2577-2588.

66. Chen J, Gao S, Wang C, Wang Z, Zhang H, Huang K, et al. Pathologically decreased expression of miR-193a contributes to metastasis by targeting WT1-E-cadherin axis in non-small cell lung cancers. J Exp Clin Cancer Res. 2016; 35(1):173.

67. Zhang JX, Zhai JF, Yang XT, Wang J. MicroRNA-132 inhibits migration, invasion and epithelial-mesenchymal transition by regulating TGF $\beta 1 / \mathrm{Smad} 2$ in human non-small cell lung cancer. Eur Rev Med Pharmacol Sci. 2016; 20(18):3793-3801.

68. Gao HX, Yan L, Li C, Zhao LM, Liu W. MiR-200c regulates crizotinib-resistant ALK-positive lung cancer cells by reversing epithelial-mesenchymal transition via targeting ZEB1. Mol Med Rep. 2016; 14(5):4135-4143.

69. Jin Z, Guan L, Song Y, Xiang GM, Chen SX, Gao B. MicroRNA-138 regulates chemoresistance in human non-small cell lung cancer via epithelial mesenchymal transition. Eur Rev Med Pharmacol Sci. 2016; 20(6):1080-1086.

70. Ye Z, Yin S, Su Z, Bai M, Zhang H, Hei Z, et al. Downregulation of miR-101 contributes to epithelial-mesenchymal transition in cisplatin resistance of NSCLCcells by targeting ROCK2. Oncotarget. 2016; 7(25): 37524-37535.

71. Li Y, Chen P, Zu L, Liu B, Wang M, Zhou Q. MicroRNA-338-3p suppresses metastasis of lung cancer cells by targeting the EMT regulator Sox4. Am J Cancer Res. 2016; 6(2):127-140.

72. Jakobsen KR, Demuth C, Madsen AT, Hussmann D, Vad-Nielsen J, Nielsen $\mathrm{AL}$, et al. MET amplification and epithelial-to-mesenchymal transition exist as parallel resistance mechanisms in erlotinib-resistant, EGFR-mutated, NSCLC HCC827 cells. Oncogenesis. 2017; 6(4):e307.

73. Yao YH, Cui Y, Qiu XN, Zhang LZ, Zhang W, Li H, et al. Attenuated LKB1-SIK1 signaling promotes epithelial-mesenchymal transition and radioresistance of non-small cell lung cancer cells. Chin J Cancer. 2016; 35: 50.

74. Kang J, Kim E, Kim W, Seong KM, Youn H, Kim JW, et al. Rhamnetin and cirsiliol induce radiosensitization and inhibition of epithelial-mesenchymal transition (EMT) by miR-34a-mediated suppression of Notch-1 expression in non-small cell lung cancer cell lines. J Biol Chem. 2013; 288(38): 27343-27357.

75. Shintani Y, Okimura A, Sato K, Nakagiri T, Kadota Y, Inoue M, et al. Epithelial to mesenchymal transition is a determinant of sensitivity to chemoradiotherapy in non-small cell lung cancer. Ann Thorac Surg. 2011; 92(5): 1794-1804

76. Tania M, Khan MA, Fu J. Epithelial to mesenchymal transition inducing transcription factors and metastatic cancer. Tumour Biol. 2014; 35(8): 7335-7342.

77. Engelman JA, Zejnullahu K, Mitsudomi T, Song Y, Hyland C, Park JO, et al. MET amplification leads to gefitinib resistance in lung cancer by activating ERBB3 signaling. Science. 2007; 316(5827):1039-1043.

78. Garofalo M, Romano G, Di Leva G, Nuovo G, Jeon YJ, Ngankeu A, et al. EGFR and MET receptor tyrosine kinase-altered microRNA expression induces tumorigenesis and gefitinib resistance in lung cancers. Nat Med. 2011; 18(1):74-82.
79. Hu $Z$, Chen $X$, Zhao $Y$, Tian $T$, Jin $G$, Shu $Y$, et al Serum microRNA signatures identified in a genome-wide serum microRNA expression profiling predict survival of non-small-cell lung cancer. J Clin Oncol.2010; 28(10): 1721-1726.

80. Lu C, Shan Z, Hong J, Yang L. MicroRNA-92a promotes epithelialmesenchymal transition through activation of PTEN/PI3K/AKT signaling pathway in non-small cell lung cancer metastasis. Int J Oncol. 2017; 51(1): 235-244.

81. Li X, Han J, Zhu H, Peng L, Chen Z. MiR-181b-5p mediates TGF- $\beta 1$-induced epithelial-to-mesenchymal transition in non-small cell lung cancer stem-like cells derived from lung adenocarcinoma A549 cells. Int J Oncol. 2017; 51(1):158-168.

82. Simone NL, Soule BP, Ly D, Saleh AD, Savage JE, Degraff W, et al. Ionizing radiation-induced oxidative stress alters miRNA expression. PLoS One. 2009; 4(7):e6377.

83. Seike M, Goto A, Okano T, Bowman ED, Schetter AJ, Horikawa I, et al. MiR-21 is

an EGFR-regulated anti-apoptotic factor in lung cancer in never-smokers. Proc Natl Acad Sci U S A. 2009; 106(29): 12085-12090.

84. Calin GA, Sevignani C, Dumitru CD, Hyslop T, Noch E, Yendamuri S, et al. Human microRNA genes are frequently located at fragile sites and genomic regions involved in cancers. Proc Natl Acad Sci U S A. 2004; 101(9): 2999-3004. 